\title{
Experimental induction of tenuazonic acid toxicity in mice model
}

\section{Ankita Kumari ${ }^{1}$ and Karuna Singh", Department of Zoology, MMV, Banaras Hindu University, India}

PP2.056

Objective- This study aims to assess the health risks associated with tenuazonic acid (TeA) produced by Alternaria alternata in tomato. In this regard a murine model has been developed to study the mycotoxicosis caused by TeA.

Material and methods- TeA was used for the experimental induction of mycotoxicosis in murine model. The mycotoxin was administered intra peritoneally (IP) and orally. Haematological, histopathological and biochemical aspects of the TeA induced toxicosis were analysed.

\section{Results-}

Behavioral observations- A significant decrease in the consumption of feed in both oral and IP groups were noted. Reductions in weights of the mice were noted in the IP group. However, an increase in the weights of the mice was recorded in the oral group.

Morphological observations- Hair loss indicating mycotoxicosis was observed in the experimental mice of both the groups.

Anatomical observations- Spleenomegaly, hepatomegaly and gross lesions on liver and kidney were noted on autopsy in both the groups. Likewise, body:organ weight indices of liver and spleen also increased .

Haematological analysis- Neutrophilia was observed in IP while neutropenia was observed in the oral group.

Biochemical analyses- Elevated malondialdehyde (MDA), reduced catalase (CAT) and superoxide dismutase (SOD) production; abnormal levels of aspartate transaminase (AST) and alanine transaminase (ALT) were observed which signifies TeA induced oxidative stress.

Histological observations- Histopathological changes characterised by non alcoholic fatty liver, nuclear pyknosis and WBC infiltration were observed.
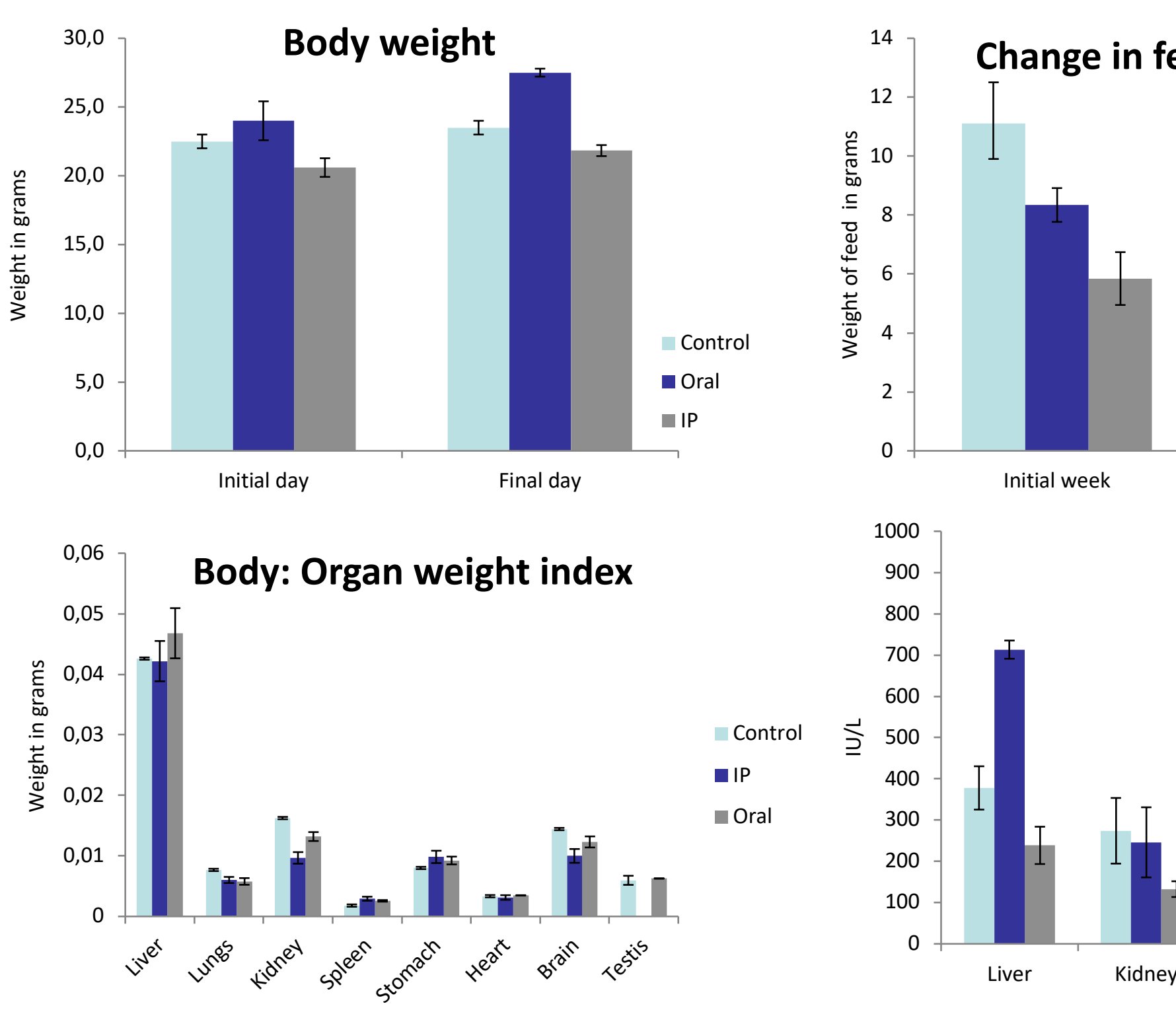

Conclusion- The findings of the above study suggest that even at a very small concentration and short exposure, TeA can cause severe damage to the vital organs. Since tomato and its by-products are used worldwide, consumption rate of TeA is high. Regulations should be framed to ensure the verification of compliance of maximum level of TeA with food and feed law. New risk assessment approaches should be considered to investigate the toxicological interactions of TeA in agricultural products, in humans and in animals.

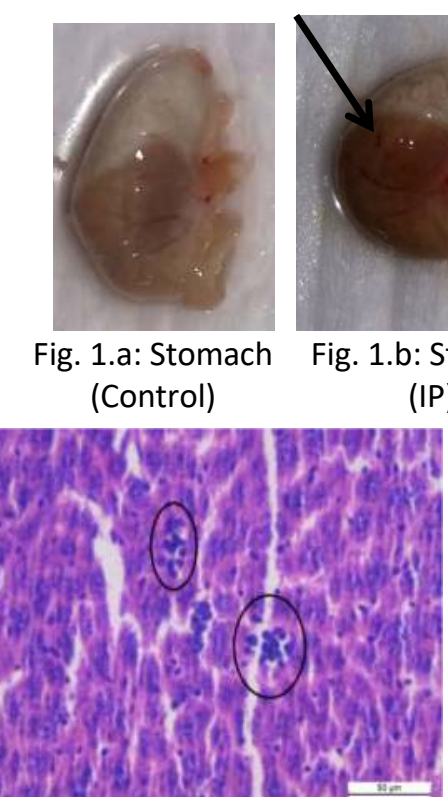

Fig. 2.d: TS. (HE $X$ 400) liver section showing necrotic liver sectio.

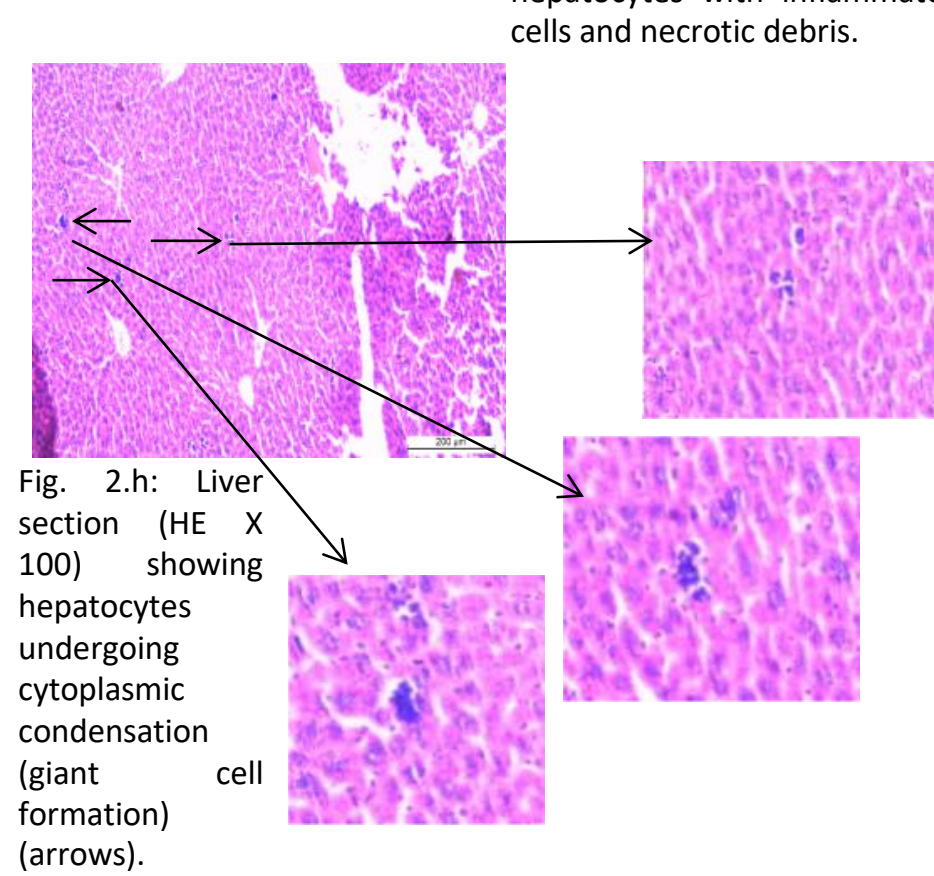
(arrows).

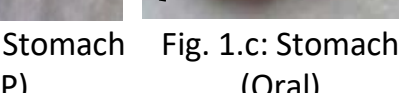

$\begin{array}{ll}\text { (IP) } & \text { (Oral) }\end{array}$

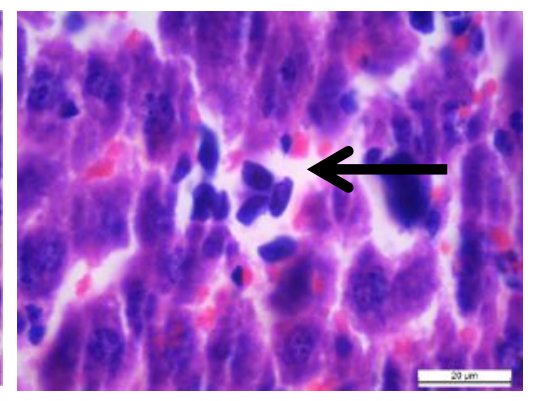

Fig. 2.e: T.S. (HE X 1000) of liver section showing a microabscess (circle) involving a few cells and necrotic debris.

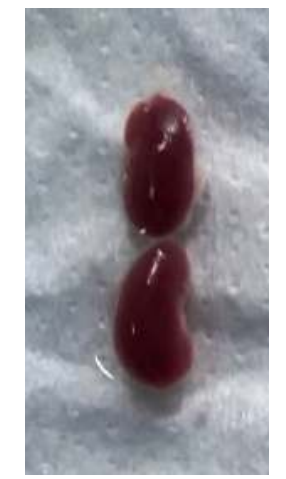

Fig. 3.a: Kidney (Control)
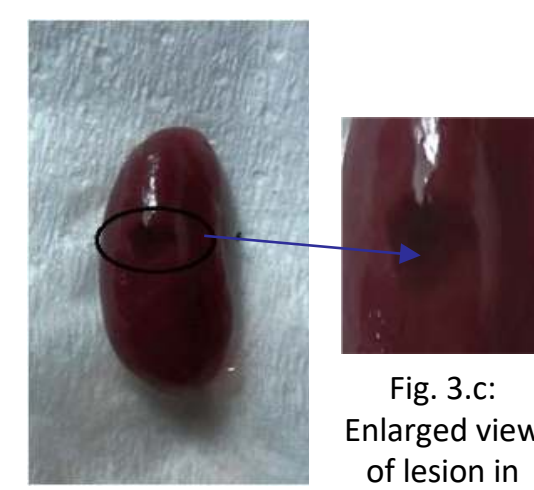

Fig. 3.b: Kidney (IP)

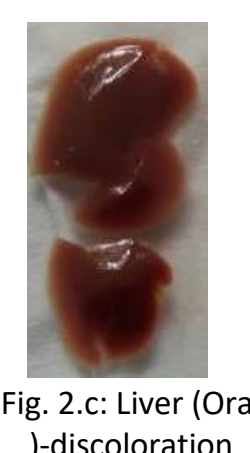
lesions

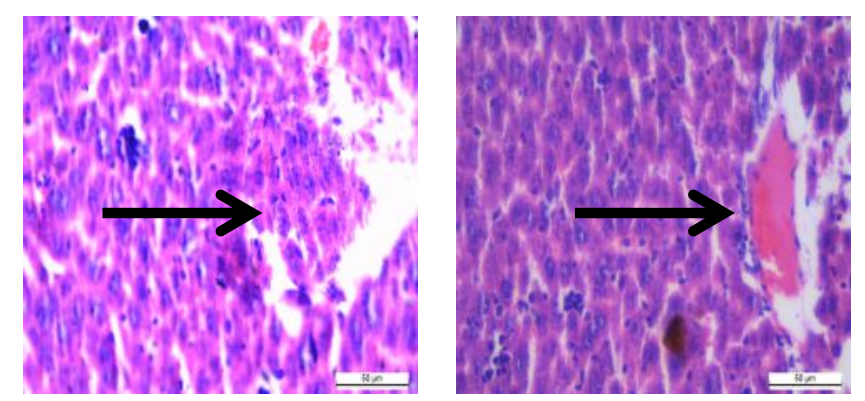

Fig. 2.f. T.S. of liver (HE X Fig. 2.g. T.S. of liver (HE X 400) showing a focal area of 400) showing a colloid

Weight gain
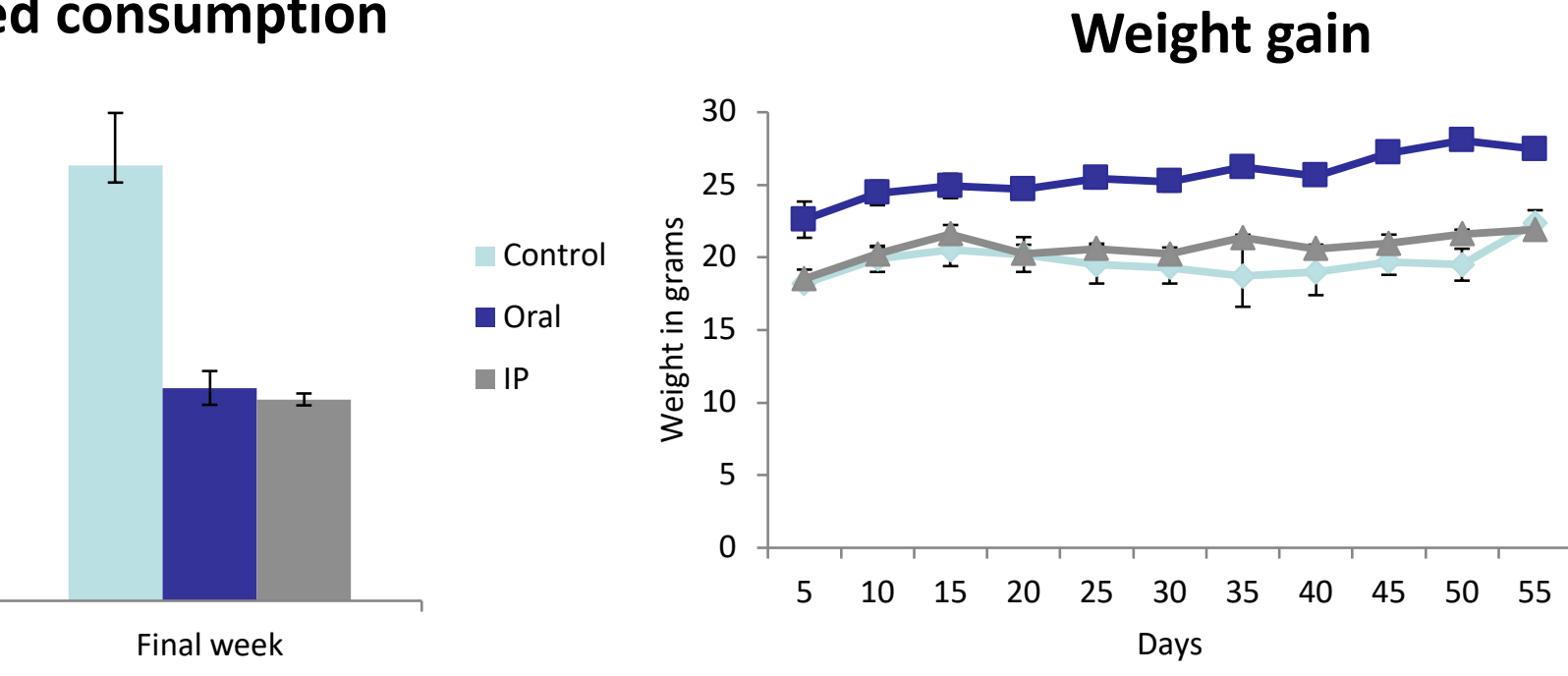

- Control
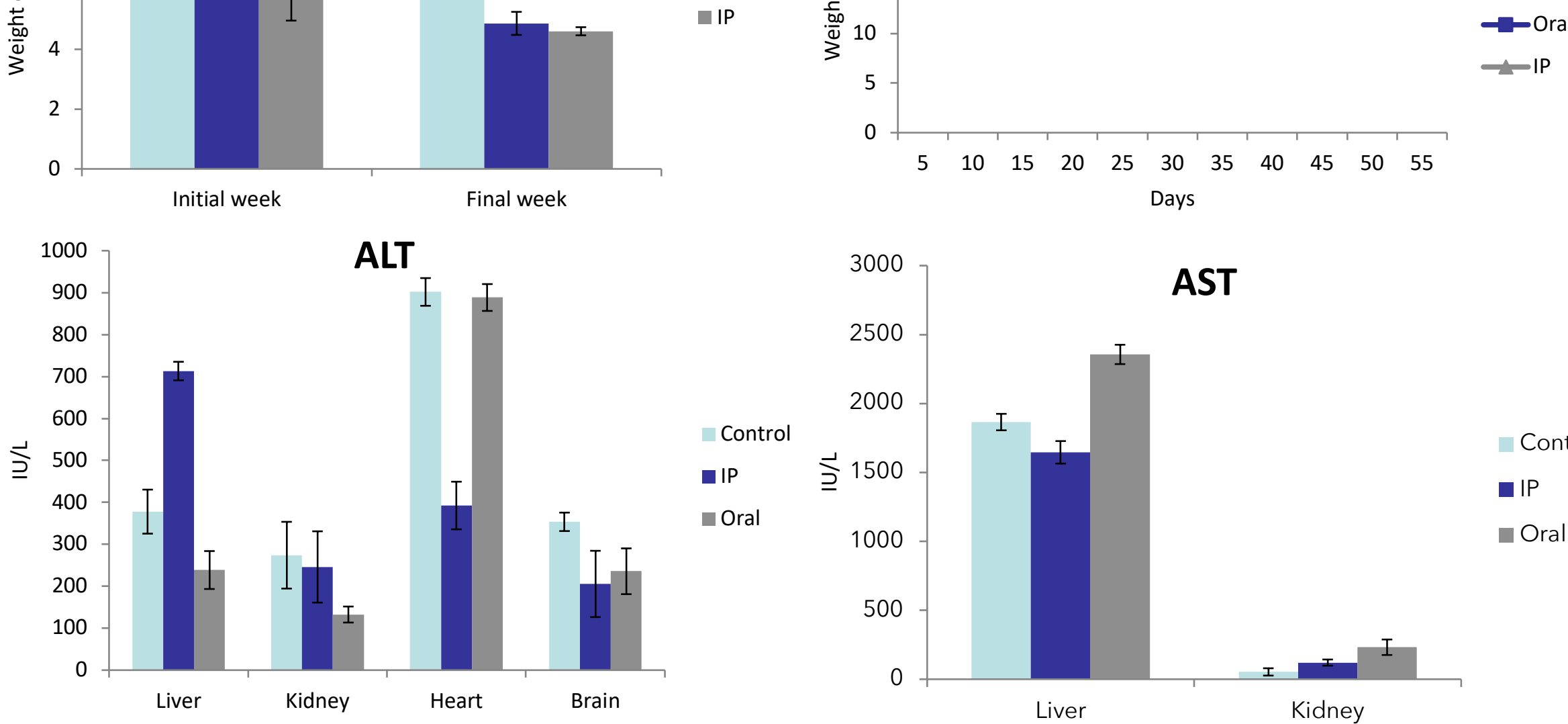

Control

- IP

- Oral

(1)

SOD

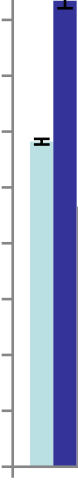

$$
\text { , }
$$

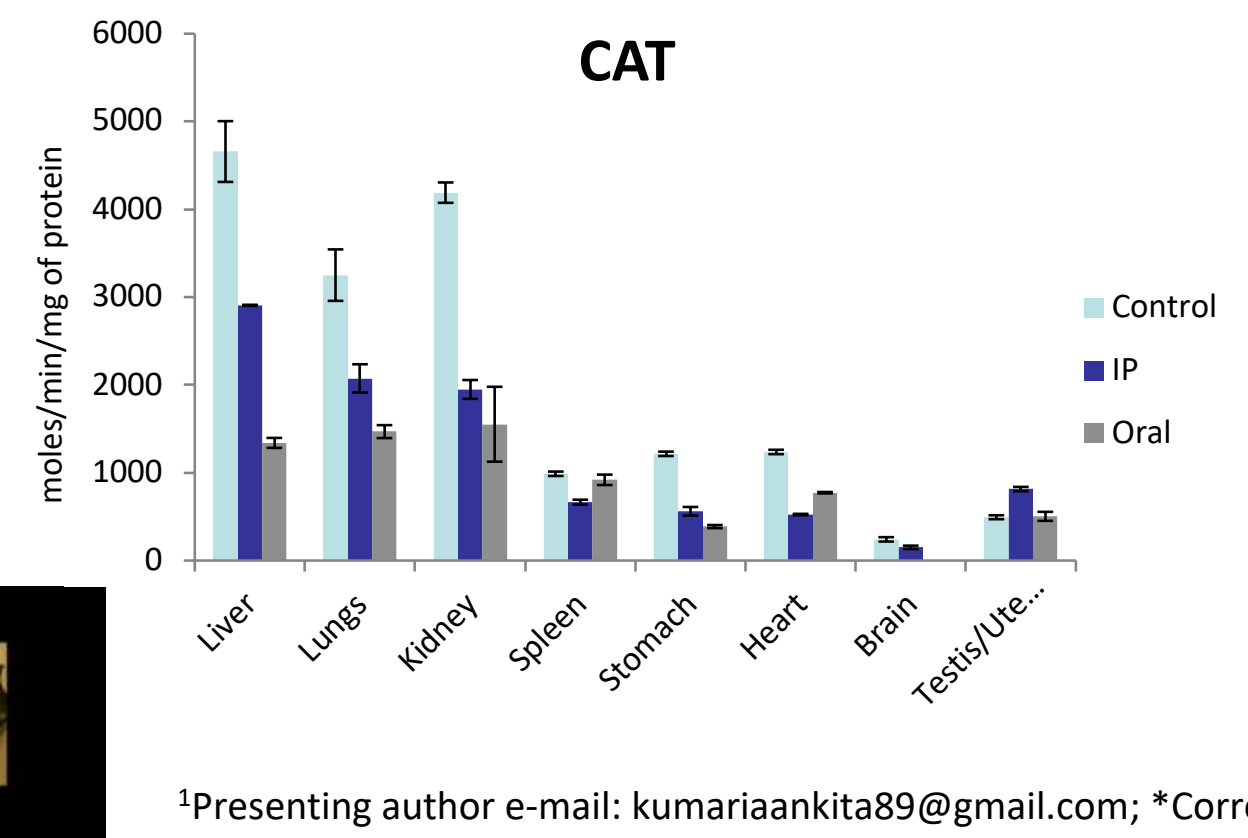

CAT

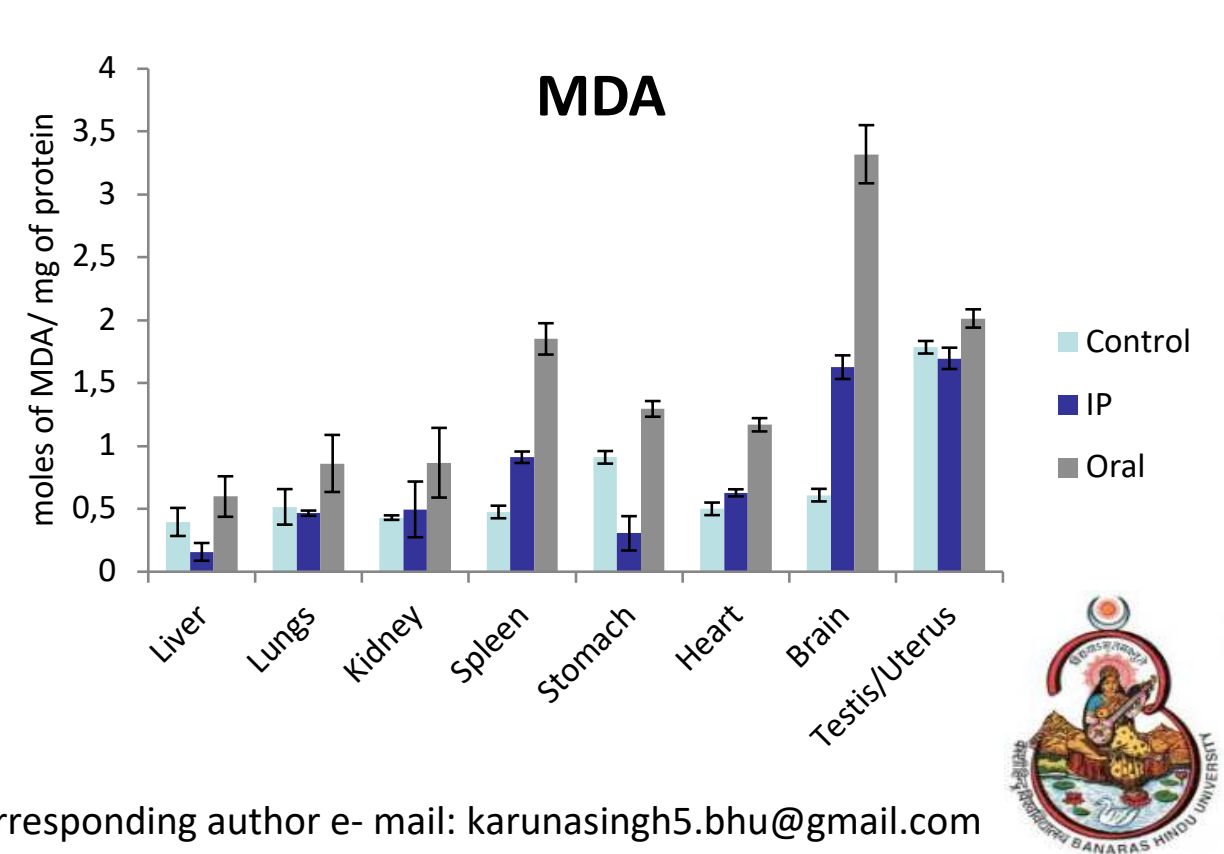

